\title{
A COMPARISON OF UWB AND MOTION CAPTURE UAV INDOOR POSITIONING
}

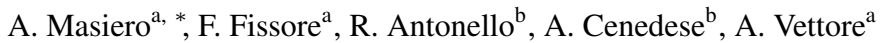 \\ ${ }^{\text {a }}$ Interdepartmental Research Center of Geomatics (CIRGEO), University of Padova, \\ Viale dell'Università 16, Legnaro (PD) 35020, Italy - \\ masiero@dei.unipd.it \\ (francesca.fissore, antonio.vettore)@unipd.it \\ ${ }^{\mathrm{b}}$ Department of Information Engineering (DEI), University of Padova, \\ via Gradenigo 6/B, Padova 35131, Italy - \\ (angelo.cenedese, riccardo.antonello)@unipd.it
}

Commission I, WG I/7

KEY WORDS: Indoor positioning, UAV, UWB, Motion Capture, Extended Kalman Filter

\begin{abstract}
:
The number of applications involving unmanned aerial vehicles (UAVs) grew dramatically during the last decade. Despite such incredible success, the use of drones is still quite limited in GNSS denied environment: indeed, the availability of a reliable GNSS estimates of the drone position is still fundamental in order to enable most of the UAV applications. Given such motivations, in this paper an alternative positioning system for UAVs, based on low cost ultra-wideband band (UWB) is considered. More specifically, this work aims at assessing the positioning accuracy of UWB-based positioning thanks to the comparison with positions provided by a motion capture (MoCap) system. Since the MoCap accuracy is much higher than that of the UWB system, it can be safely used as a reference trajectory for the validation of UWB estimates. In the considered experiment the UWB system allowed to obtain a root mean square error of $39.4 \mathrm{~cm}$ in 3D positioning based on the use of an adaptive extended Kalman filter, where the measurement noise covariance was adaptively estimated.
\end{abstract}

\section{INTRODUCTION}

Thanks to the recent worldwide spread of drones and unmanned aerial vehicle (UAV) applications (Baiocchi et al., 2018, Aicardi et al., 2016 Tucci et al., 2019), the drone market dramatically increased at a surprisingly fast rate in the last decade.

Most of the currently developed UAV applications take advantage of the GNSS positioning system in order ensure the safe and effective completion of UAV flight missions. Despite the availability and reliability of GNSS positioning system is usually ensured in most of outdoor applications in open spaces, such condition can be restrictive in certain operating conditions, in particular indoors or relatively close to high buildings, where the unreliability of the GNSS system would prevent a safe unmanned flight.

The quest for extending the usability of UAVs in such critical operating conditions is stimulating the development of new ubiquitous navigation, i.e. able to provide reliable vehicle position estimates in any working case.

Most of the solutions proposed in the literature foresee the integration of the inertial navigation system (INS) with information provided by other sensors, such as cameras radar (Mostafa et al., 2018), (Hrabar, Sukhatme, 2004), ultrasonic, LiDAR and Ultra-Wide Band (UWB) systems (Tiemann et al., 2015, Zahran et al., 2018). Some of such systems, such as vision and LiDAR systems, in some cases can be used for both navigation and mapping, which is for example the simultaneous localization and mapping (SLAM) case (Whelan et al., 2016). Despite nowadays SLAM is widely used, in

\footnotetext{
${ }^{*}$ Corresponding author.
}

particular in robotics, the usage of such sensors that enable also mapping typically lead to higher computational burdens with respect to positioning only sensors. This can be a limitation in particular in the case of small and cheap UAVs, which are typically provided with a hardware system supporting just a quite limited computational power.

Motivated by the above considerations, among the available systems that can be used for UAV positioning in indoor environments, this work deals in particular with the use of UWB positioning devices. UWB devices are radio transmitters/receivers typically characterized by the low energy transmission over a large bandwidth, i.e. larger than 500 $\mathrm{MHz}$ (Kshetrimayum, 2009). UWB devices are usually small, lightweight, low power consuming, and, their signal can be used for quite accurate precise range measurements in a wide range of working conditions. To be more specific, range measurements between UWB devices is typically obtained by means of time-of-flight measures (Gezici et al., 2005. Hol, 2011), which clearly ensure their best accuracy in clear-line-of-sight working conditions, i.e. when the line connecting the two currently ranging devices is not obstructed by any obstacle.

Despite certain more flexible configurations can be considered as well (Koppanyi et al., 2018), a set of UWB devices, hereafter named anchors, is commonly fixed at known positions. Then, the goal of the UWB positioning system is typically that of providing real-time estimates of the positions of some UWB moving devices, hereafter called rovers (or tags), exploiting the ranging measurements obtained by the anchors. A proper calibration of the anchor UWB network infrastructure can also be considered in order to improve the positioning system performance (Perakis, Gikas, 2018, Toth et al., 2015). 
Exploiting the current ranging measurements between rover and anchors, the position of moving UWB rovers can be estimated by solving a trilateration geometric problem. Actually, since the rover position should be tracked in real time, the position estimates are often obtained by suitable implementations of the Extended Kalman filter (Goel et al., 2017).

According to the results reported in several recently published research papers, the positioning accuracy of UWB systems is expected to be at decimeter level, however, there are quite limited studies on their positioning performance in the case of UAVs used in indoor environments, probably mostly due to the difficulty of accurately measuring the real time position of UAVs in such working conditions.

In order to assess the performance of a UWB system in UAV indoor positioning, this work presents a comparison between position estimates obtained by means of UWB devices with those provided by a Motion Capture (MoCap) system.

The comparison is made in an indoor environment, in a $105 \mathrm{~m}^{3}$ flying arena. Since the MoCap system provides position estimates with a much higher accuracy with respect to UWB, its estimated trajectory is used as reference in order to validate UWB one.

The rest of the paper is organized as follows: Section 2 describes the used UWB and MoCap systems. Section 3 presents the considered approach, based on the use of an adaptive Extended Kalman filter (Pointon et al., 2019, Akhlaghi et al., 2017 Ding et al., 2007), for obtaining UAV position estimates by means of UWB measurements. Then, the obtained results are shown in Section 4 and, finally, some conclusions are drawn in Section 5 .

\section{SYSTEM DESCRIPTION}

In this work a Pozyx UWB rover is attached to a drone (Figure 1 a)) and used to track the drone position thanks to the range measurements provided by a set of four Pozyx UWB anchors distributed in the room (Figure 1 b)).

Each Pozyx device is a quite low cost $(\$ 150$ per device, approximately) UWB radio transmitter/receiver. Pozyx UWB devices are very portable, and in particular they are low weight and small, which are very interesting characteristics in order to use them in UAV applications. The maximum range is approximately $100 \mathrm{~m}$, which can be sufficient for enabling UAV UWB-based positioning in not too much large areas. The random part of the Pozyx UWB ranging error is relatively small, whereas the systematic part is affected by several factors (Masiero et al., 2017), and, in particular, it is environment dependent (e.g. multi-path issues).

The drone position was simultaneously tracked also by a Motion Capture BTS Smart-D system, composed by 12 photogrammetric IR cameras. Once properly calibrated, such system provided drone position estimates at up to $100 \mathrm{~Hz}$ (60 $\mathrm{Hz}$ in the considered case study), with a high position accuracy (nominal accuracy better than $1 \mathrm{~mm}$ ), by means of robust triangulation algorithms (Masiero, Cenedese, 2012).

The flying arena (Fig. 11 measures $7 \times 5 \times 3 \mathrm{~m}^{3}$ and the 12 cameras are distributed on a rig around the perimeter so as to minimize occlusions during normal flying conditions of more UAVs and guarantee a reconstruction contribution of more than eight cameras in all the available volume.

Given the very high accuracy of the MoCap system, in this work its estimates are used as reference trajectory to be compared with that provided by the UWB devices.

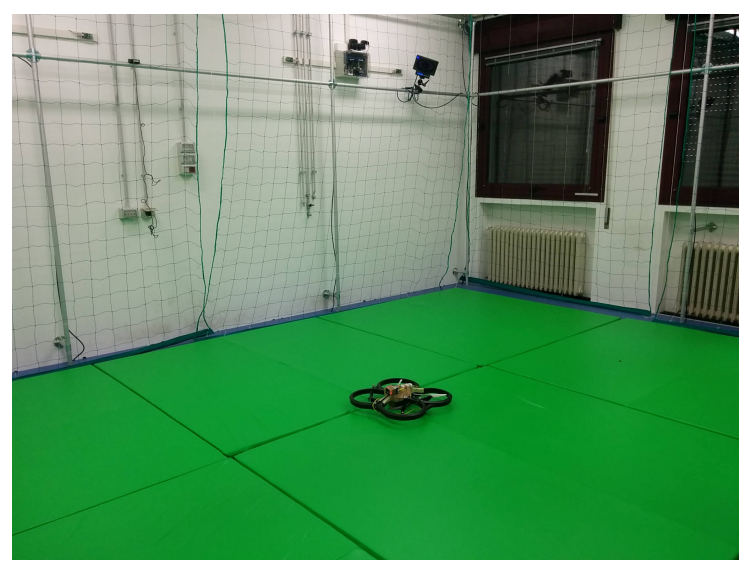

(a)

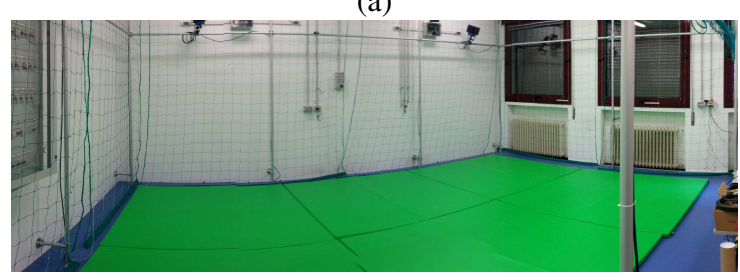

(b)

Figure 1. The considered UAV (a), and a panoramic view of the flying arena (b).

\section{POSITION ESTIMATIONS BASED ON UWB MEASUREMENTS}

The UWB range measurement equation can be expressed as follows:

$$
y_{i, t}=\left\|q_{i}-p_{t}\right\|+v_{i, t}
$$

where $\quad y_{i, t}=$ range measured at time $t$ between rover and anchor $i$

$p_{t}=$ rover position at time $t$

$q_{i}=$ position of the $i$-th anchor

$v_{i, t}=$ measurement error on $y_{i, t}$

$v_{i, t}$ is composed by a systematic and by a random part, and, in practice, it is influenced by several factors, such as the UWB device synchronization error, multi-path issues.

The UWB rover position is obtained by means of an extended Kalman filter (EKF), where the linearized version of (1) is used as measurement equation.

The rover dynamic is modeled as follows:

$$
x_{t+\Delta t}=A x_{t}+w_{t}
$$


where

$$
\begin{aligned}
& x_{t}=\left[\begin{array}{c}
p_{t} \\
\dot{p}_{t}
\end{array}\right] \\
& \dot{p}_{t}=\text { rover velocity at time } t \\
& A=\left[\begin{array}{cc}
I & \Delta t^{2} I \\
0 & I
\end{array}\right] \\
& I=\text { identity matrix } \\
& w_{t}=\text { zero-mean random noise with covariance } \\
& \qquad t^{2} Q
\end{aligned}
$$

It is worth to notice that the UWB range measurement from the available anchors are collected at different time instants by the rover: in the considered implementation, the Pozyx rover iteratively try to communicate with each of the anchors in a loop, and, a range measurement is stored after each rover-anchor interaction, if available. The time delay between two received range measurements is in the considered system approximately $0.02 \mathrm{~s}$, whereas ranges from the $i$-th anchor (for any value of $i$ ) are checked approximately every $0.1 \mathrm{~s}$ seconds.

Consequently, at least two alternative options can be considered for the implementation of the EKF:

A) Each range measurement can be processed in the EKF just after its availability.

B) All range measurements in the same loop are processed together. It is worth to notice that, despite this is not the case of the considered implementation, if at least four ranges are available a 3D position can also be computed without considering prior information about the rover position (and about its dynamic).

Furthermore, two different options are considered for the range measurement noise (which in both the cases is assumed to be a zero-mean process): use a constant (pre-set) value for the variance of the range measurement noise, or adapt its covariance matrix according to the real system measurements. The second approach, which is similar to those presented in (Pointon et al., 2019, Akhlaghi et al., 2017. Ding et al., 2007), clearly allows a better flexibility and adaptability to the current working conditions. In particular, the measurement noise covariance shall be updated (for instance using a proper forgetting factor (Akhlaghi et al., 2017)) by means of the residuals $\epsilon$ :

$$
\epsilon_{t, i}=y_{t, i}-\left\|q_{i}-\hat{p}_{t \mid t}\right\|
$$

where $\hat{p}_{t \mid t}$ is the rover position at time $t$ estimated by the EKF by using the measurement $y_{t, i}$.

In practice, the following four EKF cases, obtained by combining the alternative options mentioned above, are considered in the following section:

- EKF1: processing each UWB range measurement when it is available with pre-set constant measurement noise.

- Adapt.EKF1: the same as EKF1, but with measurement variance noise adaptively estimated (separately for each anchor).

- EKF2: processing UWB range measurements at the end of each loop, i.e. when new measurements from all anchors can also be available. Noise covariance is constant.

- Adapt.EKF2: the same as EKF2, but with measurement covariance noise adaptively estimated.

\section{RESULTS}

In this section, the considered EKF approaches for computing UWB-based UAV position estimates are compared with those obtained by the MoCap system

The comparison is done on a $300 \mathrm{~s}$ flight, whose reference trajectory, provided by the MoCap, is shown in Fig. 2

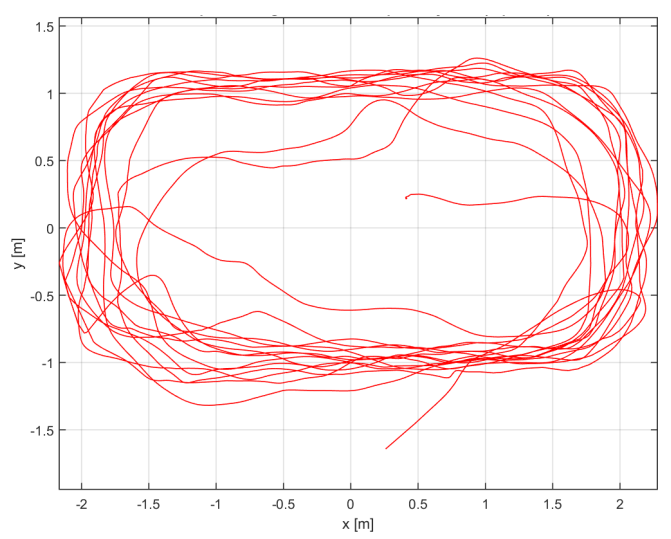

Figure 2. UAV position tracked by the MoCap system.

Fig. 3 shows a comparison of the reference UAV trajectory with that obtained by means of the UAV system (in the EKF1 case).

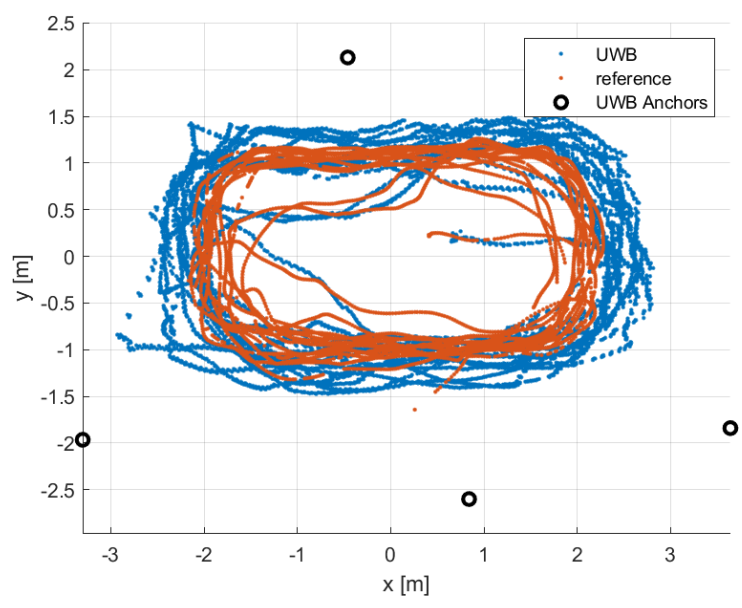

Figure 3. Comparison of UWB estimated positions (blue) with reference ones (red).

The root mean square error (for the 3D position estimates, for the horizontal and vertical position error) and the maximum (absolute) error along the $x$ and $y$ directions are reported in the Table 1 for all the considered cases.

\begin{tabular}{|l|c|c|c|c|c|}
\hline \hline & $\begin{array}{c}\text { RMS } \\
\text { 3D }\end{array}$ & $\begin{array}{c}\text { RMS } \\
\text { 2D }\end{array}$ & $\begin{array}{c}\text { RMS } \\
z\end{array}$ & $\begin{array}{c}\max \\
\text { err } x\end{array}$ & $\begin{array}{c}\max \\
\text { err } y\end{array}$ \\
\hline \hline & & & & & \\
EKF1 & 47.1 & 34.3 & 32.3 & 74.5 & 76.2 \\
Adapt.EKF1 & 42.0 & 32.4 & 26.8 & 78.1 & 95.7 \\
EKF2 & 42.7 & 30.3 & 30.0 & 82.7 & 69.6 \\
Adapt.EKF2 & 39.4 & 28.9 & 26.8 & 86.5 & 87.5 \\
& & & & & \\
\hline
\end{tabular}

Table 1. UWB positioning errors $[\mathrm{cm}]$.

Furthermore, Fig. 4, 5, 6 and 7, show the estimated position errors along the three directions for all the considered cases. 


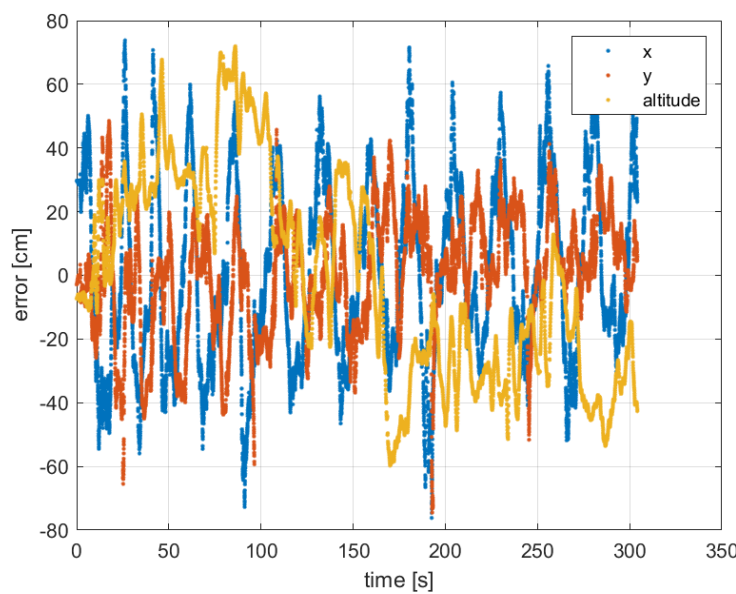

Figure 4. Errors on the UWB position estimates in the EKF1 case.

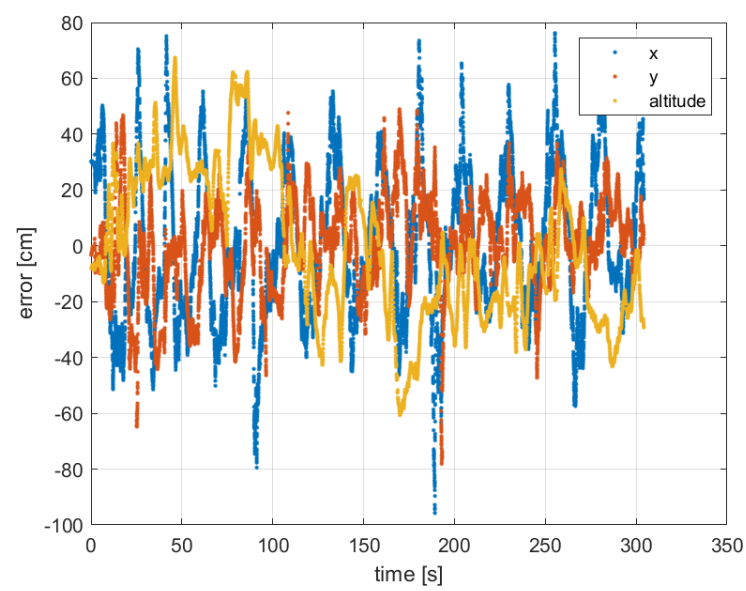

Figure 5. Errors on the UWB position estimates in the Adapt.EKF1 case.

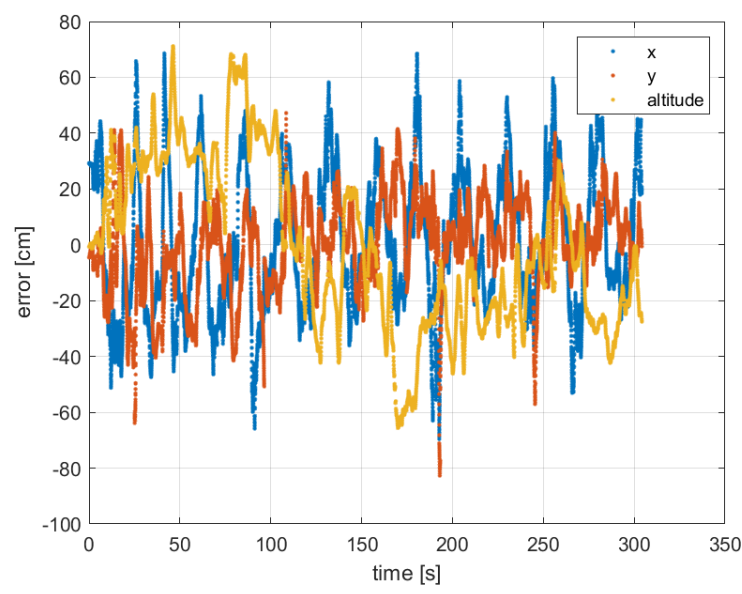

Figure 6. Errors on the UWB position estimates in the EKF2 case.

Finally, similarly to the GPS case, Fig. 8 shows the horizontal dilution of precision (HDOP) according to the geometry of the considered UWB positioning system (evaluated at $1.5 \mathrm{~m}$ of altitude with respect to the ground, which is half of the height of the flying arena).

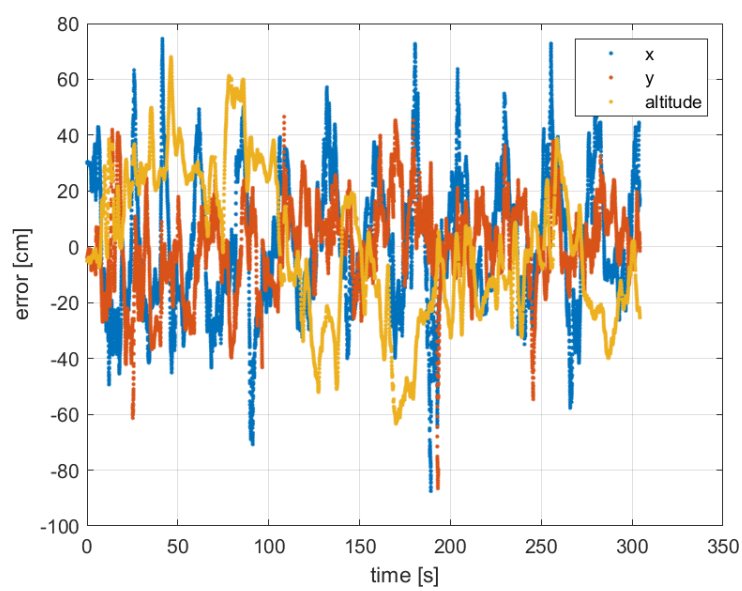

Figure 7. Errors on the UWB position estimates in the Adapt.EKF2 case.

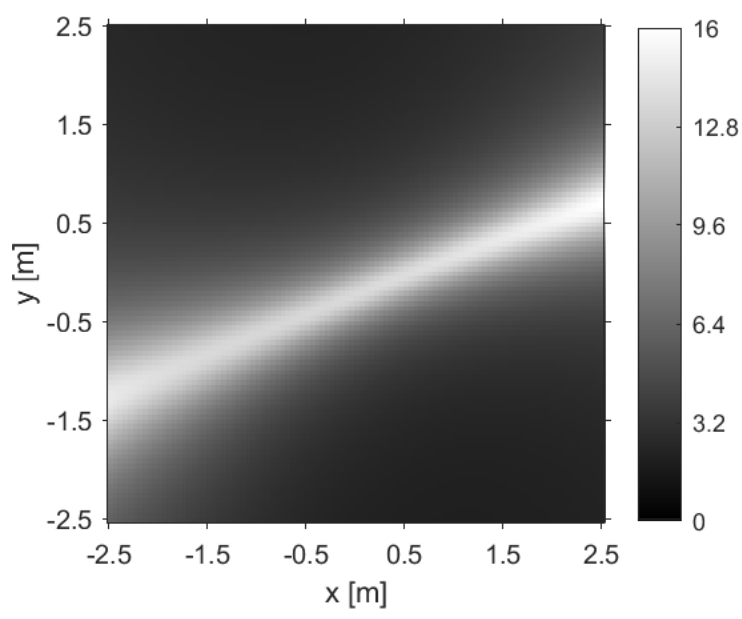

Figure 8. HDOP.

\section{CONCLUSIONS}

The results obtained in the considered experiment show that the use of an adaptive measurement noise covariance allows to slightly improve the performance of the UWB positioning system.

Processing each UWB measurement just after its arrival allows to take into account of the different time of arrivals of the different range measurements: despite should take to some advantages, in practice, processing together the ranges available after checking in a loop from all the anchors allowed in the considered case study to obtain better results. In such case the different times of arrival of the range measurements have been discarded, however, since the maximum velocity used in such small indoor environment has been quite limited (approximately $2 \mathrm{~m} / \mathrm{s}$ on the rectilinear parts of the trajectory, with few exceptions at higher velocities), such approximation led to a relatively small error in this experiment. When the UAV can reach higher velocities (e.g. in larger areas/rooms) taking into consideration of the different time of availability of the range measurements (such as in EKF1 and Adapt.EKF1) may lead to some advantage.

Maximum errors in the considered experiment are typically close to UAV changes of the moving direction, and associated 
to relatively large measurement errors, i.e. when both the UAV dynamic and range measurements do not allow to properly deal with the change of moving direction. Actually, such errors are quite clear for instance on the bottom-left of Fig. 3 it is worth to notice that such areas is also one of those where the DOP has a higher value (Fig. 8), hence the geometric configuration of the UWB anchors is not particularly well suited for estimating the drone position in such area.

To conclude, the results obtained in the considered comparison show that adaptive EKF can be used in order to obtain UAV $3 \mathrm{D}$ position estimates in real time with accuracy (in our experiment) at $40 \mathrm{~cm}$ level $(30 \mathrm{~cm}$ for what concerns the $2 \mathrm{D}$ position).

\section{REFERENCES}

Aicardi, I., Dabove, P., Lingua, A.M., Piras, M., 2016. Integration between TLS and UAV photogrammetry techniques for forestry applications. iForest-Biogeosciences and Forestry, 10,41 .

Akhlaghi, S., Zhou, N., Huang, Z., 2017. Adaptive adjustment of noise covariance in kalman filter for dynamic state estimation. 2017 IEEE Power Energy Society General Meeting, $1-5$.

Baiocchi, V., Napoleoni, Q., Tesei, M., Costantino, D., Andria, G., Adamo, F., 2018. First tests of the altimetric and thermal accuracy of an uav landfill survey. 2018 5th IEEE International Workshop on Metrology for AeroSpace (MetroAeroSpace), IEEE, 403-406.

Ding, W., Wang, J., Rizos, C., Kinlyside, D., 2007. Improving adaptive Kalman estimation in GPS/INS integration. The Journal of Navigation, 60, 517-529.

Gezici, S., Tian, Z., Giannakis, G.B., Kobayashi, H., Molisch, A.F., Poor, H.V., Sahinoglu, Z., 2005. Localization via ultra-wideband radios: a look at positioning aspects for future sensor networks. IEEE signal processing magazine, 22, 70-84.

Goel, S., Kealy, A., Gikas, V., Retscher, G., Toth, C.s, Grejner-Brzezinska, D., Lohani, B., 2017. Cooperative Localization of Unmanned Aerial Vehicles Using GNSS, MEMS Inertial, and UWB Sensors. Journal of Surveying Engineering, 143, 04017007.

Hol, J., 2011. Sensor Fusion and Calibration of Inertial Sensors, Vision, Ultra-Wideband and GPS. PhD. Thesis, Linköping University, The Institute of Technology.

Hrabar, S., Sukhatme, G.S., 2004. A comparison of two camera configurations for optic-flow based navigation of a uav through urban canyons. IROS, 2673-2680.
Koppanyi, Z., Toth, C. K., Brzezinska, D. G., 2018. Scalable ad-hoc uwb network adjustment. 2018 IEEE/ION Position, Location and Navigation Symposium (PLANS), 1502-1508.

Kshetrimayum, R.S., 2009. An introduction to UWB communication systems. IEEE Potentials, 28, 9-13.

Masiero, A., Cenedese, A., 2012. On triangulation algorithms in large scale camera network systems. Proceedings of the 2012 American Control Conference, ACC 2012, Montréal, Canada, 4096-4101.

Masiero, A., Fissore, F., Vettore, A., 2017. A low cost UWB based solution for direct georeferencing UAV photogrammetry. Remote Sensing, 9, 414.

Mostafa, M., Zahran, S., Moussa, A., El-Sheimy, N., Sesay, A., 2018. Radar and Visual Odometry Integrated System Aided Navigation for UAVS in GNSS Denied Environment. Sensors, 18,2776 .

Perakis, H., Gikas, V., 2018. Evaluation of range error calibration models for indoor uwb positioning applications. 2018 International Conference on Indoor Positioning and Indoor Navigation (IPIN), IEEE, 206-212.

Pointon, H.A.G., McLoughlin, B.J., Matthews, C., Bezombes, F.A., 2019. Towards a Model Based Sensor Measurement Variance Input for Extended Kalman Filter State Estimation. Drones, 3.

Tiemann, J., Schweikowski, F., Wietfeld, C., 2015. Design of an UWB indoor-positioning system for UAV navigation in GNSS-denied environments. Indoor Positioning and Indoor Navigation (IPIN), 2015 International Conference on, IEEE, $1-7$.

Toth, C.K., Jozkow, G., Ostrowski, S., Grejner-Brzezinska, D., 2015. Positioning slow moving platforms by UWB technology in GPS-challenged areas. The 9th International Symposium on Mobile Mapping Technology, MMT 2015.

Tucci, G., Parisi, E.I., Castelli, G., Errico, A., Corongiu, M., Sona, G., Viviani, E., Bresci, E., Preti, F., 2019. Multi-Sensor UAV Application for Thermal Analysis on a Dry-Stone Terraced Vineyard in Rural Tuscany Landscape. ISPRS International Journal of Geo-Information, 8, 87.

Whelan, Thomas, Salas-Moreno, Renato F, Glocker, Ben, Davison, Andrew J, Leutenegger, Stefan, 2016. ElasticFusion: Real-time dense SLAM and light source estimation. The International Journal of Robotics Research, 35, 1697-1716.

Zahran, S., Mostafa, M.M., Masiero, A., Moussa, A.M., Vettore, A., El-Sheimy, N., 2018. Micro-RADAR and UWB aided UAV navigation in GNSS denied environment. ISPRS International Archives of the Photogrammetry, Remote Sensing and Spatial Information Sciences, XLII-1, 469-476. 